\title{
NeLH Oral Health Specialist Library www.nelh.nhs.uk/oralhealth - how to access evidence based material quickly
}

\author{
Ruth Turley ${ }^{a}$, Elizabeth Treasure ${ }^{\text {b }}$, Alison Weightmanc, John Potts ${ }^{\text {d }}$ \\ ${ }^{a}$ Information Officer, University of Wales College of Medicine, Heath Park, Cardiff, Wales \\ ${ }^{\mathrm{b}}$ Professor of Dental Public Health, University of Wales College of Medicine, Heath Park, Cardiff, Wales \\ ${ }^{\mathrm{c}}$ Acting Deputy Director of Library Services, University of Wales College of Medicine, Heath Park, Cardiff, \\ Wales \\ dSenior Lecturer in Oral Pathology, University of Wales College of Medicine, Heath Park, Cardiff, Wales
}

\begin{abstract}
Launched by HRH Duke of Edinburgh on 25th March 2003, the Oral Health Specialist Library of the National Electronic Library for Health (NeLH) supplies rapid access to up-to-date, relevant, reliable evidence. The Library is at the initial stage of a four year project and is one of the first to adopt the new design template that will soon come into effect for all the specialist libraries. It will continue to be developed through the work of an operation team based at the University of Wales College of Medicine, Cardiff. The group consists of oral health care professionals and an information team, advised by a UK wide steering group.

Evidence-Based Dentistry (2003) 4, 34-36. doi:10.1038/sj.ebd.6400190
\end{abstract}

The ultimate goal of the Oral Health Specialist Library is to provide a service to aid oral health workers, in improving health and healthcare. With the continuous growth in oral health literature, it has become difficult for practitioners to keep in touch with new research. The library will address this by acting as a major vehicle for dissemination, systematically gathering and assessing the quality of information to assist with, and promote the use of, evidence in practice. Knowledge-sharing will be encouraged by the provision of discussion forums and details of pending oral health events (Figure 1).

There has been widespread support for the initiative, including strong backing from the Chief Dental Officers of the country. Professor Raman Bedi, Chief Dental Officer (England) supported the NeLH with the words, "Information for patients, the public, healthcare professionals and those who manage the NHS is essential for the delivery of a quality service. The need for this information to be relevant, accurate and accessible is critical. The NeLH is an important step in helping us achieve this." His counterpart in Wales, Paul Langmaid concurs, "I am delighted to see this initiative launched in Wales. I am very supportive of it as it will provide a useful resource not only for clinicians but patients and others seeking evidence on the treatment they provide and receive."

\section{What is the NeLH?}

Since its launch 2 years ago, the National Electronic Library for Health (NeLH) has gone from strength to strength, attracting 35000 visitors per month last year (double the number in its first year). Established in response to the govern- ment's 'Information for Health' strategy, the NeLH concentrates well-respected evidence-based initiatives to provide healthcare professionals and the public with knowledge and know-how to support healthcare-related decisions.

Over 70 electronic resources, ranging from bibliographic databases such as Medline or the Cochrane Library to Reviewed Internet Sites by NMAP (Nursing, Midwifery and Allied health Professions), have been gathered. Many of these have existed for some time but were previously scattered across a range of locations. As far as possible, the NeLH is committed to making health information available to all who need access, and broke new ground when it licensed the Cochrane Library and Clinical Evidence on behalf of the National Health Service (NHS) in England. It continues to work to bring together existing and new resources into a user-friendly environment, and such a collection is unique.

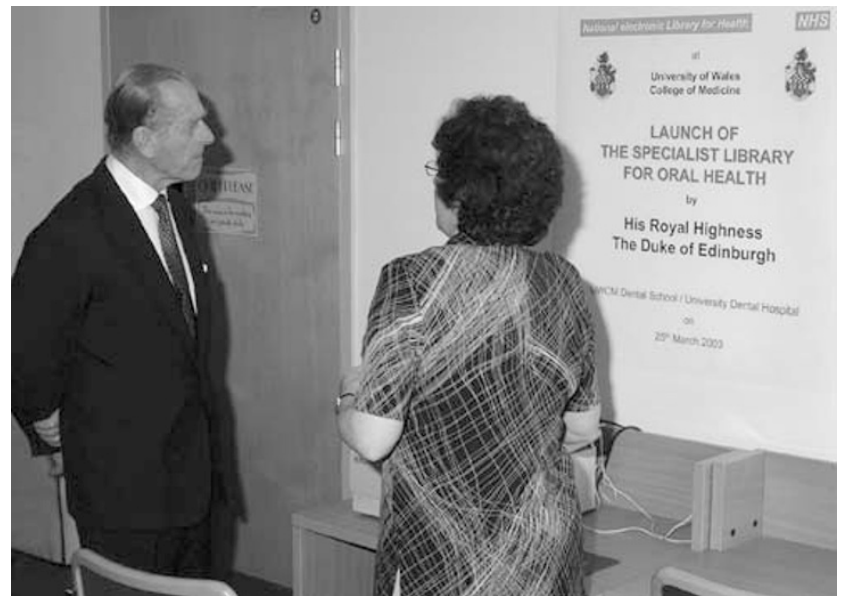

Figure 1. HRH The Duke of Edinburgh launches the Oral Health Specialist Library. 


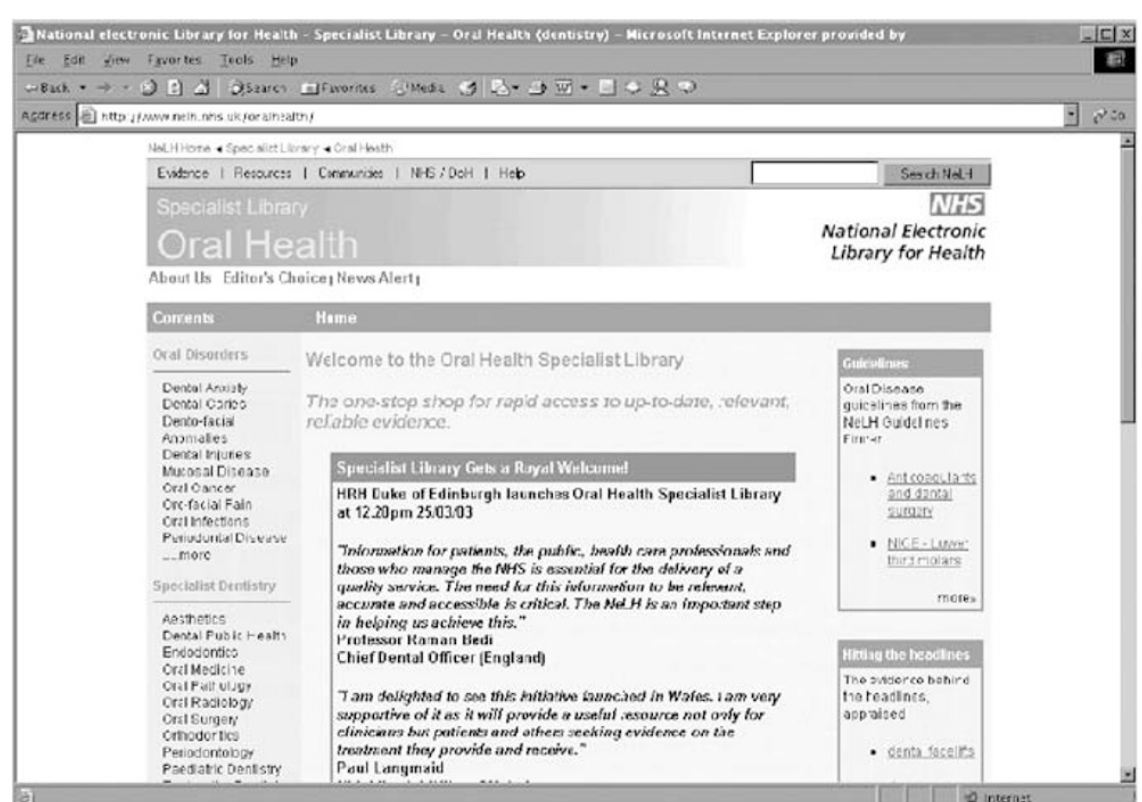

Figure 2. Oral Health Specialist Library — homepage.

A priority for the NeLH is working in partnerships and building communities of practice. To achieve this, an extensive collection of specialist electronic libraries for specific healthcare interests has been assembled. The newest addition is the Oral Health Specialist Library (Figure 2).

\section{For whom is the Oral Health Specialist Library intended?}

Oral health professionals, their trainees and students and other healthcare workers use the library, but patients and the public may also wish to view the site for information regarding their healthcare.

\section{What does the Library currently offer?} Quality assurance - With the increasing volumes of oral health literature, it has become increasingly difficult for practitioners to keep up-to-date with recent research. This is further complicated by the release of studies of varying degrees of quality. The library will function to direct its users to the best-quality research, by critically appraising all the evidence it hosts, and providing a summary of its validity. Recent, relevant information is systematically searched for and each publication included is assessed according to the library's quality policy which uses an internationally recognised method of appraisal devised by the Support Unit for Evidence Research at the University of Wales College of Medicine. Evidence is tagged with the 'resource type' (systematic review, clin- ical governance, randomised controlled trial) and a critical summary is provided of the research. Any other resources included on the site that have not been subjected to the quality policy will be clearly marked. Currently the library hosts reviewed evidence with critical summaries and links to full text reviews, on the subject of dental caries. As well as aiding practitioners, the critical summaries are useful in explaining the evidence to non-specialists (other health workers, students and trainees, librarians and the public) (Figure 3).

\section{Content and finding your way around}

\section{it}

All information relevant to oral health and healthcare will be gathered by the library. Publications are provided around two main areas of oral health:

(i) Oral disorders (including, for example, dental caries, oral cancer and periodontal disease);

(ii) Specialist dentistry (eg, aesthetics, dental public health and orthodontics); and

(iii) Quality and Safety (compiling clinical governance, care pathways and protocols, and guidelines).

A full list of contents is shown on the navigation bar, the left-hand column of each page. Evidence is accessed by selecting the relevant subject in this section. Presently, the library hosts quality-assured systematic reviews for dental caries and also provides links to relevant oral health guidelines, which will be critically appraised and summaries provided in due course. The library will be regularly updated, and work is under way to fully populate all areas of the contents bar. Also listed in the contents are links to key organisations such as the dental council, relevant royal colleges, the Centre for Evidence-based Dentistry and the Cochrane Library. A link to details of pending events such as congresses and courses is also available in addition to a feedback form to collect user opinion.

\section{Dentistry in the news}

Can dental facelifts make you look younger? Does passive smoking cause dental caries? Can toothpaste made from crabshells fight tooth decay? These are some of the dentistry headlines featured in the news recently, although medical research is not always reported accurately. "Dentistry in the News" sets out to examine the real evidence behind selected national newspaper and media reports to provide information about their reliability and answer any patient questions generated by such articles. Unbiased, comprehensive summaries appraising the research evidence are posted on the library in a similar format to "Hitting the Headlines" featured on the main NeLH site, which covers a broader range of health issues.

\section{Links to the main NeLH site}

To ensure links to the main NeLH site are maintained, and that other appropriate medical research is made readily accessible, a toolbar heading the Oral Health Specialist Library contains links to the following NeLH sections:

Evidence (eg, guidelines, systematic reviews);

Resources, communities (linking to other specialist libraries and profession portals); NHS/Department of Health links; and the NeLH search engine.

\section{The library is under development -} what can be expected in the future?

Search engine: A search engine will be put in place so that appropriate information can be located rapidly. Search results will be separated into types of evidence and sorted by date within these groups. Therefore users will be able to choose quickly the best and most recent quality information for their search question.

Discussion forum: A discussion forum will be provided to encourage the devel- 


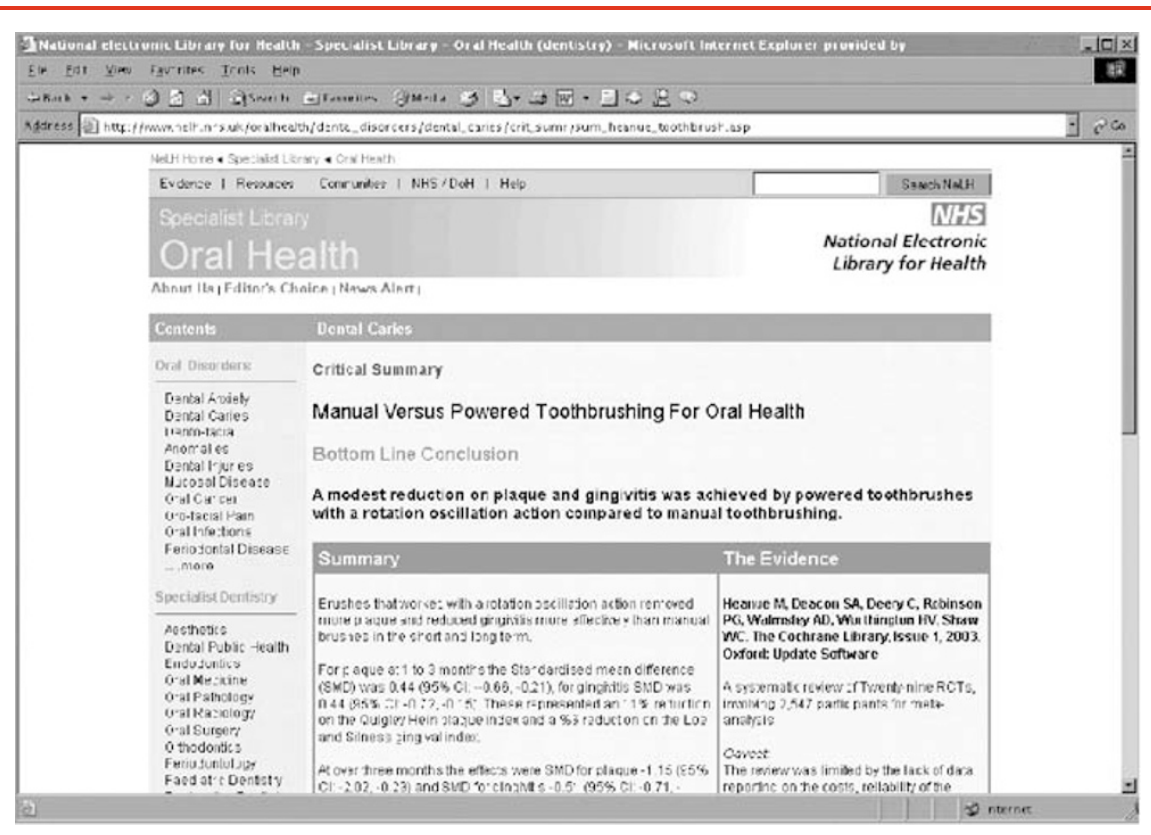

Figure 3. Oral Health Specialist Library - critical summary page.

opment of communities of practice. Users will be able to build key contacts and informally exchange ideas, offering and sharing their expertise to others whilst widening their own knowledge beyond their specific speciality.

Quality and safety: This section supplies all relevant clinical pathways and proto- cols, guidelines and clinical governance, so that users have access to and are kept informed with the know-how and resources needed to deliver consistent and advanced patient-centred health care.

Continual professional development (CPD): This scheme aims to invite users to disseminate papers according to the library's quality policy, and submit their appraisals and critical summaries, in exchange for CPD points. This will serve several purposes: users can keep in touch with recent evidence at their own pace, hone critical appraisal skills, and help to develop and populate the library that serves them.

Extensive population: All the topics within dental disorders and specialist dentistry will be populated with resource references and critical summaries. Guidelines and systematic reviews will be sought and disseminated first, followed shortly by clinical governance, clinical pathways, and randomised controlled trials and other study types. An information officer will be working on the library full time, and so the content will be extended and updated on a regular basis.

\section{How do I get involved?}

Your views on the Oral Health Specialist Library are welcomed, so please explore www.nelh.co.uk/oralhealth and let us know what sort of things you would like to see on the site. Follow the links on the site to post your feedback as we aim to be responsive to its users. 